\title{
The incidence of deep vein thrombosis detected by routine surveillance ultrasound in neurosurgery patients receiving dual modality prophylaxis.
}

\author{
Patricia C Henwood \\ Brigham and Women's Hospital, Boston MA \\ Thomas M Kennedy \\ Thomas Jefferson University \\ Lynda Thomson \\ Thomas Jefferson University \\ Follow this and additional works at: https://jdc.jefferson.edu/petfp \\ Taki Galanis

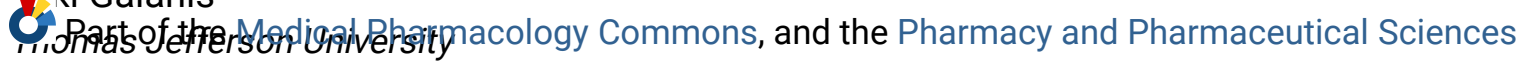 \\ Commons

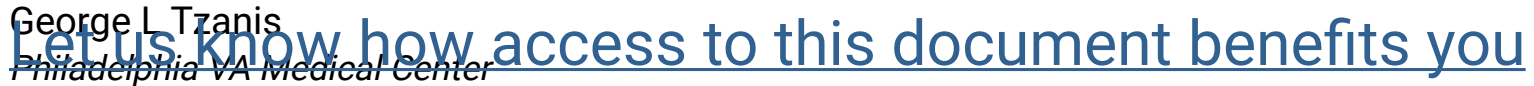

Recommended Citation

See next page for additional authors

Merli, Geno J; and Kraft, Walter K., "The incidence of deep vein thrombosis detected by routine surveillance ultrasound in neurosurgery patients receiving dual modality prophylaxis." (2011). Department of Pharmacology and Experimental Therapeutics Faculty Papers. Paper 25. https://jdc.jefferson.edu/petfp/25

This Article is brought to you for free and open access by the Jefferson Digital Commons. The Jefferson Digital Commons is a service of Thomas Jefferson University's Center for Teaching and Learning (CTL). The Commons is a showcase for Jefferson books and journals, peer-reviewed scholarly publications, unique historical collections from the University archives, and teaching tools. The Jefferson Digital Commons allows researchers and interested readers anywhere in the world to learn about and keep up to date with Jefferson scholarship. This article has been accepted for inclusion in Department of Pharmacology and Experimental Therapeutics Faculty Papers by an authorized administrator of the Jefferson Digital Commons. For more information, please contact: JeffersonDigitalCommons@jefferson.edu. 


\section{Authors}

Patricia C Henwood, Thomas M Kennedy, Lynda Thomson, Taki Galanis, George L Tzanis, Geno J Merli, and Walter K. Kraft 


\title{
As submitted to: \\ Journal of Thrombosis and Thrombolysis
}

And later published as:

\section{The incidence of deep vein thrombosis detected by routine surveillance ultrasound in neurosurgery patients receiving dual modality prophylaxis}

\author{
Volume 32, Issue 2, August 2011, Pages 209-214
}

\section{DOI: 10.1007/s11239-011-0583-8}

Patricia C. Henwood, MD ${ }^{\sharp t}$, Thomas M. Kennedy, BS*, Lynda Thomson, PharmD*, Taki Galanis, MD*, George Tzanis, $\mathrm{MD}^{\S}$, Geno J. Merli, MD*, Walter K. Kraft, MD*

"DDepartment of Emergency Medicine, Brigham and Women's Hospital, Boston, MA 02115

*Jefferson Vascular Center, Thomas Jefferson University, Philadelphia, PA 19107

${ }^{\S}$ Department of Internal Medicine, Veterans Affairs Medical Center, Philadelphia, PA 19104

Corresponding Author:

Patricia C. Henwood, MD

Department of Emergency Medicine

Brigham and Women's Hospital

75 Francis St., Neville House

Boston, MA 02115

Phone 610-952-1050

phenwood@partners.org

Word Count: 2369

Abstract Word Count: 223

References: 20

Tables: 3

Figures: 1

Keywords : neurosurgery, ultrasound, deep vein thrombosis, prophylaxis, screening

\author{
Abbreviations: \\ ACCP $=$ American College of Chest Physicians. \\ $\mathrm{DVT}=$ deep vein thrombosis \\ $\mathrm{ICH}=$ intracranial hemorrhage \\ $\mathrm{JHN}=$ Jefferson Hospital for Neuroscience \\ $\mathrm{LMWH}=$ low molecular weight heparin
}


$\mathrm{PE}=$ pulmonary embolism

$\mathrm{U}=$ units

UFH = unfractionated heparin

VTE $=$ venous thromboembolism 


\begin{abstract}
The optimal method of thromboprophylaxis and the value of screening ultrasonography for detection of deep venous thrombosis (DVT) in neurosurgery patients remains unclear. The goal of this study was to determine the incidence of DVT in neurosurgical patients who, by hospital protocol, receive surveillance ultrasonography of the lower extremities twice weekly, in addition to prophylaxis with unfractionated heparin and external pneumatic compression sleeves. A retrospective review of 7,298 ultrasound studies carried out on 2,593 patients over four years at a university neurosurgical hospital was conducted. There was a $7.4 \%$ incidence of proximal lower extremity DVT and a $9.7 \%$ total incidence including distal DVT. A greater number of distal DVTs were detected with the implementation of whole-leg ultrasonography in the last two years of observation. Chart review of 237 patients diagnosed with DVT demonstrated an admitting diagnosis of subarachnoid hemorrhage in nearly half of the patients. The median hospital length of stay for DVT patients was 18 days. Institutional control data demonstrated non-ruptured aneurysm and cerebrovascular anomalies to be the leading reason for admission, followed closely by subarachnoid hemorrhage. The hospital protocol of biweekly screening ultrasound and dual modality prophylaxis for neurosurgery patients resulted in a proximal DVT incidence consistent with that demonstrated by previous studies of standardized dual modality prophylaxis, and higher than that demonstrated in previous studies that employed ultrasound screening protocols.
\end{abstract}




\section{Introduction}

Venous thromboembolism (VTE) is a diagnostic term that encompasses deep vein thrombosis (DVT) and its most serious complication, pulmonary embolism (PE). Best estimates are that 350,000 to 600,000 Americans develop VTE each year, with PE causing 180,000 deaths annually [1]. Indeed, PE is the number one cause of preventable death in hospitalized patients [2]. There are many published guidelines for VTE prophylaxis, of which those from the American College of Chest Physicians (ACCP) are the most widely used [3]. Yet, only 62\% of patients undergoing major surgical procedures at risk for VTE are receiving ACCP-recommended prophylaxis globally [4]. The high incidence of hospital-acquired VTE demonstrates the current gap between evidence-based prophylaxis guidelines and quality measure implementation [5].

Patients undergoing neurosurgery are considered to be at moderate risk for VTE [2], with the rate of DVT for combined cranial/spinal procedures performed without prophylaxis varying from 29 to 43\% [6]. However, patients with spinal cord injury, malignancy, increasing age, cardiac or respiratory failure, prolonged immobility, and a wide variety of inherited and acquired hematologic conditions are at considerably higher risk [7]. The optimal method of VTE prophylaxis in neurosurgical patients is a matter of controversy. The landmark 1998 study by Agnelli et al. demonstrated the superiority of combined pharmacologic and mechanical prophylaxis over mechanical methods alone in neurosurgical patients, reducing the risk of VTE from 33 to 17\% [8]. Several subsequent studies have similarly reported lower rates of VTE with multimodality prophylaxis $[9,10]$. However, some neurosurgeons do not use heparin for peri-procedural prophylaxis secondary to concerns of significant bleeding complications [11].

In 2005, Danish et al. compared the safety and efficacy of pharmacologic anticoagulants with mechanical prophylaxis for craniotomy patients using decision analysis computer modeling to simulate virtual trials based on statistics from published literature [12]. Modeling suggested that the addition of heparin lowered the incidence of both DVT and PE, but at the cost of increasing the rate of intracranial hemorrhage (ICH). The authors concluded that the deleterious effects of ICH are greater than the benefits from pharmacologic anticoagulation on VTE morbidity and mortality, and that overall outcomes are best with mechanical prophylaxis alone. A meta-analysis conducted in 2008 support the findings by Danish et al [13].

The current ACCP guidelines recommend that mechanical prophylaxis, using intermittent pneumatic compression devices, be used in neurosurgical patients [2]. Acceptable alternatives to intermittent pneumatic compression devices include postoperative low molecular weight heparin (LMWH) or perioperative low dose 
unfractionated heparin. For patients who have a particularly high risk of thrombosis, combined mechanical and pharmacologic methods are recommended.

Another area of debate in VTE prophylaxis is surveillance ultrasonography for postoperative neurosurgery patients. The evidence base upon with which standardized DVT surveillance protocols or guidelines can be created is lacking. The natural history of incidentally discovered, asymptomatic DVT is unclear. It is possible that these have a lower propensity to propagate or embolize, and that aggressive treatment in a high bleeding-risk population may provide more harm than benefit. The question of the correct frequency of surveillance screening in neurosurgical patients, or if it should be performed at all, remains unanswered.

The objective of the current study was to retrospectively assess the incidence of lower extremity DVT, as diagnosed by surveillance compression ultrasonography (US), in the setting of a dual modality prophylaxis regimen aimed at minimizing VTE among neurosurgical patients at the Jefferson Hospital for Neuroscience (JHN) over a four year time period.

JHN is a free-standing university neurosurgical specialty hospital where the standard of care is for patients who are not actively being treated for DVT to have surveillance ultrasound twice weekly, regardless of their clinical status, mobility, diagnosis, or symptoms. In addition, the standard of care calls for all neurosurgical patients to receive, within twenty-four hours of hospitalization, prophylaxis with external pneumatic compression sleeves and unfractionated heparin (UFH) 5000 units (U) every 12 hours.

\section{Methods}

A retrospective review of ultrasound database records for all neurosurgical patients admitted who had compression ultrasound surveillance of the lower extremity during the four year study period, from January 2003 and December 2006, was completed. Total number of lower extremity ultrasound studies performed as well as positivity for lower extremity DVT was collected. Distal (calf vein) versus proximal (popliteal, branches of femoral, or external iliac vein) location of the DVT was recorded.

Prior to 2005, the distal lower extremity was not routinely examined during surveillance ultrasound. In the last two years of the data collection period, a change to the standard ultrasound technique in was made to include the entire lower extremity, capturing images from the iliac to calf veins and thereby allowing for detection of distal 
DVT. All upper extremity ultrasound studies were excluded from review. Each patient was counted only once for DVT occurrence during the entire study period, regardless of their number of positive ultrasound scans.

Electronic chart review was possible for 237 of 252 patients identified as positive for lower extremity DVT over the four year study period. Data was collected on dates of admission and discharge, as well as primary diagnosis, for these 237 patientsAt the time of this study, the medical records in our system were partially computerized and partially paper. As a result, collection of more comprehensive data on each patient was limited. Administrative hospital data was used to estimate an approximate case mix of all patients admitted to the neurosurgical services.

\section{Results}

A total of 7,298 ultrasound studies performed on the lower extremities of 2,593 patients were eligible for analysis (Table 1). Of these patients, 252 (9.7\%) were found to have proximal and/or distal lower extremity DVT (Figure 1; Table 2). The number of patients with proximal lower extremity DVT alone was 193 (7.4\%). The age distribution of patients with DVT was 18 to 97 years, with a mean age of 61 . Of DVT patients, $42 \%$ were male and $58 \%$ were female. Three distal DVTs were detected in 2003-04, while 56 were detected in the 2005-06. While direct case controls without thrombosis were not available due to the nature of the database search method used, hospital-wide census data revealed a mean patient age of approximately 56 years old.

For 237 patients identified with DVT, subarachnoid hemorrhage was the leading primary diagnosis, accounting for $48.5 \%$ of cases. ICH was the reason for admission in $11.8 \%$ of DVT patients, $11 \%$ were admitted with malignancy, and $8.9 \%$ with non-ruptured cerebral aneurysm and cerebrovascular anomalies (Table 3). Among the general neurosurgical population admitted to JHN, the approximate case mix revealed non-ruptured cerebral aneurysm and cerebrovascular anomalies accounted for approximately $30 \%$ of admissions, followed by $24 \% \mathrm{SAH}$, $8 \% \mathrm{ICH}$ and $7 \%$ malignancy. The DVT population in this study had a larger proportion of patients admitted with SAH, ICH or malignancy as primary diagnosis, and significantly fewer patients admitted with non-ruptured aneurysms, when compared to hospital-wide data.

The median length of stay for DVT patients reviewed was 18 days. There was a trend toward decreasing length of stay observed over the four year time period. Compared to these hospital-wide patient data, DVT patients had longer lengths of stay regardless of primary diagnosis. Most notably, patients with non-ruptured cerebral 
aneurysms and cerebrovascular anomalies who developed DVTs had longer lengths of stay than other patients with the same diagnosis.

\section{Discussion}

The reported incidence of DVT in neurosurgical patients varies widely. The biweekly screening protocol revealed an overall incidence of $7.4 \%$ proximal lower extremity DVT and $9.7 \%$ total lower extremity DVT in neurosurgical patients where the hospital protocol was to administer prophylaxis with UFH 5,000 U twice daily and external pneumatic compression sleeves within 24 hours of admission.

The proximal DVT rate in our study is consistent with data published from a number of other studies. Nurmohamed et al. examined the incidence of both proximal and total lower extremity DVT in neurosurgical patients. Patients in that study received LMWH (nadroparin) and graduated compression stockings, and by venography were found to have a proximal lower extremity incidence of 7\%; however, the incidence of total lower extremity DVT was considerably higher at 19\% [14]. Similarly, Agnelli et al. demonstrated an incidence of 5\% proximal and 17\% total lower extremity DVT, in patients treated with both LMWH (enoxaparin) and compression stockings as detected by venography at treatment day 8 (or sooner if the patient developed symptoms of DVT confirmed by compression ultrasound) [8]. Both of these studies revealed a significant reduction in the rate of DVT when combined prophylactic regimens were employed. In comparison, our study demonstrated a much smaller proportion of distal DVTs detected. Whole-leg ultrasound was not practiced in the first two years of our investigation which contributed to lower overall rates of distal DVT. The lower percentage of distal DVT may also be due, in part, to decreased sensitivity of ultrasound when compared to venography employed in the above studies. It is also possible that operator variability, technically limited calf studies, or a learning curve related to change in ultrasound procedure led to decreased detection of calf vein DVT in 2005 and 2006, when whole leg ultrasonography was being practiced.

In 2002, Goldhaber et al. used a multimodality prophylactic regimen of enoxaparin $40 \mathrm{mg} / \mathrm{dl}$ or UFH 5,000 U twice daily, graduated compression stockings, intermittent pneumatic compression stockings, and one predischarge venous ultrasound study of the legs in 150 craniotomy patients [10]. This approach resulted in no patients with symptomatic DVT, but a total lower extremity asymptomatic DVT incidence of $9.3 \%$, very similar to our $9.7 \%$ rate of total lower extremity DVT. We were unable to determine symptomatic vs. asymptomatic DVT as our 
investigation was a retrospective review of an ultrasound database, and documentation in the charts reviewed was such that it was not able to be clearly determined. Based on our population and prior studies, we assume the large majority were asymptomatic and detected by the biweekly screening protocol.

Depending on the severity of their condition, many neurosurgical patients may not exhibit signs or express symptoms of thrombosis, so surveillance ultrasound may play a unique role in the early detection of DVT and prevention of $\mathrm{PE}$ in this patient population. However, there is conflicting data in the literature regarding propagation rates and long-term clinical significance of distal (calf vein) DVT and the appropriate management of these cases [15-17]. Screening ultrasound in neurosurgery patients increases diagnosis of asymptomatic calf vein DVT and furthers the debate on appropriate management of these patients at increased risk for intracranial hemorrhage. Hence, it is clear that more large scale prospective studies are needed to help address this issue.

In a similar fashion to our described prophylaxis and surveillance protocol, Misra et al. reviewed routine ultrasound performed twice weekly on 1,094 patients admitted to a neurosurgical intensive care unit [18]. All of these patients were administered DVT prophylaxis in the form of UFH $5000 \mathrm{U}$ twice daily and sequential compression boots at all times until patients were able to ambulate. The incidence of DVT was $2.55 \%$ compared to $5.62 \%$ in patients of all other ICUs within the same hospital that did not adhere to a strict prophylactic regimen or screening protocol. Furthermore, there was a very low progression of DVT to PE in the neurosurgical intensive care patients (3.6\%) compared to patients in all other intensive care units (19\%), suggesting that the neurosurgical intensive care bi-weekly screening protocol helped in the early detection of DVT and prevention of PE. Compared to these studies, the incidence of DVT in our study is higher. This may be related to the larger examined patient population in our study, or possibly a larger proportion of high-risk patients.

Whether it is cost-effective to be screening all neurosurgical patients twice weekly is still unclear. This type of screening protocol employed by Misra et al. was estimated to cost between $\$ 1,300$ to $\$ 1,800$ per week. ${ }^{18}$ It may be advantageous to follow a screening protocol such as this in only those neurosurgical patients at particularly high-risk. For example, a study of 2,643 neurosurgical patients demonstrated a 7.7\% incidence of DVT with routine screening in patients undergoing cranial surgery, but only a $1.5 \%$ incidence in spinal surgery patients [19]. The authors suggested that only craniotomy patients require routine screening. Similarly, Ray et al. identified patients who suffered aneurysmal subarachnoid hemorrhage as being a high-risk group for DVT development, having a $24 \%$ incidence of asymptomatic DVT [20]. The benefit of routine screening in these high-risk groups, and others like 
them, likely outweighs concerns of cost-effectiveness. Our results indicate that SAH is one of the most common primary diagnoses at our institution, and in the vast majority of those patients identified with DVT in this study, hence we feel this patient population is generally high-risk, favoring the prophylaxis and surveillance ultrasonography protocol we employ.

There are a number of limitations to this investigation, including its retrospective nature. Mortality, bleeding complication rates, incidence of PE, and the number of diagnostic studies for PE ordered in patients with DVT were not obtained due to the nature of the database employed. Other limitations include an unknown number of patients therapeutically anticoagulated at presentation, or with a prior history of DVT. Patients received a standardized therapy, and there was no control group against which to compare management approaches. Furthermore, the standard of care at JHN calls for all neurosurgical patients to receive, within twenty-four hours of hospitalization, prophylaxis with external pneumatic compression sleeves and UFH 5000 units every 12 hours, but it is possible that deviation from this prophylaxis protocol impacted our rates of DVT.

Obtaining individual demographic information for those patients screened and found to be negative for DVT was beyond the scope of our study. Accordingly, a separate database, which included cases and controls, was used to establish general trends among all patients admitted to JHN for a period of time during our study. Finally, the case mix of this hospital is unique in that it is a free-standing neurosurgical hospital specializing in aneurysm and SAH management, so the generalizability of our data may be limited.

In conclusion, the biweekly screening protocol and dual modality prophylaxis regimen employed in this large neurosurgical population of 2,593 patients over four years, dominated by patients with cerebral aneurysms and SAH, yielded a total DVT incidence of 9.7\%. Proximal DVT incidence of $7.4 \%$ is consistent with that demonstrated by previous studies of standardized dual modality prophylaxis, and higher than that demonstrated in previous studies additionally employing routine screening ultrasound protocols. The use of screening ultrasonography protocols is

still debated, and further investigation into whether it is useful and cost-effective to performing screening ultrasounds routinely or in selected high-risk groups within the neurosurgical population is warranted.

\section{Acknowledgements}

The authors would like to thank Daniel Louis and Megan Templin for assistance in obtaining hospital data. 


\section{References}

1. The Surgeon General's Call to Action to Prevent Deep Vein Thrombosis and Pulmonary Embolism. U.S. Department of Health and Human Services [Internet] 2008 [cited 2010 Nov 6]. Available from: http://www.surgeongeneral.gov/topics/deepvein/calltoaction/call-to-action-on-dvt-2008.pdf

2. Geerts WH, Bergqvist D, Pineo GF, et al (2008) Prevention of venous thromboembolism: American College of Chest Physicians Evidence-Based Clinical Practice Guidelines (8th Edition). Chest 133(6 Suppl): 381S-453S.

3. Hirsh J, Guyatt G, Albers GW, Harrington R, Schunemann HJ, American College of Chest Physician (2008) Antithrombotic and thrombolytic therapy: American College of Chest Physicians Evidence-Based Clinical Practice Guidelines (8th Edition). Chest 133(6 Suppl): 110S-112S.

4. Kakkar AK, Cohen AT, Tapson VF, et al (2010) Venous thromboembolism risk and prophylaxis in the acute care hospital setting (ENDORSE survey): findings in surgical patients. Ann Surg 251(2): 330-338.

5. Deitelzweig SB, Lin J, Hussein M, Battleman D (2009) Are surgical patients at risk of venous thromboembolism currently meeting the Surgical Care Improvement Project performance measure for appropriate and timely prophylaxis? J Thromb Thrombolysis 30(1): 55-66.

6. Black PM, Baker MF, Snook CP (1986) Experience with external pneumatic calf compression in neurology and neurosurgery. Neurosurgery 18(4): 440-444.

7. Anderson FA,Jr, Spencer FA (2003) Risk factors for venous thromboembolism. Circulation 107(23 Suppl 1):I916.

8. Agnelli G, Piovella F, Buoncristiani P, et al (1998). Enoxaparin plus compression stockings compared with compression stockings alone in the prevention of venous thromboembolism after elective neurosurgery. $\mathrm{N}$ Engl J Med 339(2): 80-85.

9. Iorio A, Agnelli G (2000) Low-molecular-weight and unfractionated heparin for prevention of venous thromboembolism in neurosurgery: a meta-analysis. Arch Intern Med 160(15): 2327-2332.

10. Goldhaber SZ, Dunn K, Gerhard-Herman M, Park JK, Black PM (2002) Low rate of venous thromboembolism after craniotomy for brain tumor using multimodality prophylaxis. Chest 122(6): 1933-1937.

11. Gnanalingham KK, Holland JP (2003) Attitudes to the use of prophylaxis for thrombo-embolism in neurosurgical patients. J Clin Neurosci 10(4): 467-469.

12. Danish SF, Burnett MG, Ong JG, Sonnad SS, Maloney-Wilensky E, Stein SC (2005) Prophylaxis for deep venous thrombosis in craniotomy patients: a decision analysis. Neurosurgery 56(6): 1286-1294.

13. Collen JF, Jackson JL, Shorr AF, Moores LK (2008) Prevention of venous thromboembolism in neurosurgery: a metaanalysis. Chest 134(2): 237-249.

14. Nurmohamed MT, van Riel AM, Henkens CM, et al (1996) Low molecular weight heparin and compression stockings in the prevention of venous thromboembolism in neurosurgery. Thromb Haemost 75(2): 233-238. 
15. Bernardi E, Camporese G, Buller HR, et al (2008) Serial 2-point ultrasonography plus D-dimer vs whole-leg color-coded Doppler ultrasonography for diagnosing suspected symptomatic deep vein thrombosis: a randomized controlled trial. JAMA 300(14): 1653-1659.

16. MacDonald PS, Kahn SR, Miller N, Obrand D (2003) Short-term natural history of isolated gastrocnemius and soleal vein thrombosis. J Vasc Surg 37:523-527.

17. Gillet JL, Perrin MR, Allaert FA (2007) Short-term and mid-term outcome of isolated symptomatic muscular calf vein thrombosis. J Vasc Surg 46:513-519.

18. Misra M, Roitberg B, Ebersole K, Charbel FT (2004) Prevention of pulmonary embolism by combined modalities of thromboprophylaxis and intensive surveillance protocol. Neurosurgery 54 (5):1099-1103.

19. Flinn WR, Sandager GP, Silva MB,Jr, Benjamin ME, Cerullo LJ, Taylor M (1996) Prospective surveillance for perioperative venous thrombosis: experience in 2643 patients. Arch Surg 131(5):472-480.

20. Ray WZ, Strom RG, Blackburn SL, Ashley WW, Sicard GA, Rich KM (2009) Incidence of deep venous thrombosis after subarachnoid hemorrhage. J Neurosurg 110(5):1010-1014. 
Table 1 Compression ultrasound studies performed

\begin{tabular}{|l|c|c|c|c|c|}
\hline & $\mathbf{2 0 0 3}$ & $\mathbf{2 0 0 4}$ & $\mathbf{2 0 0 5}$ & $\mathbf{2 0 0 6}$ & \multicolumn{1}{c|}{ Total } \\
\hline Total lower extremity studies & 1786 & 1905 & 1878 & 1729 & 7298 \\
\hline Patients with lower extremity studies & 505 & 625 & 747 & 716 & 2593 \\
\hline Mean studies per patient & 3.5 & 3.1 & 2.5 & 2.4 & 2.8 \\
\hline
\end{tabular}


Figure 1 Incidence of lower extremity DVT

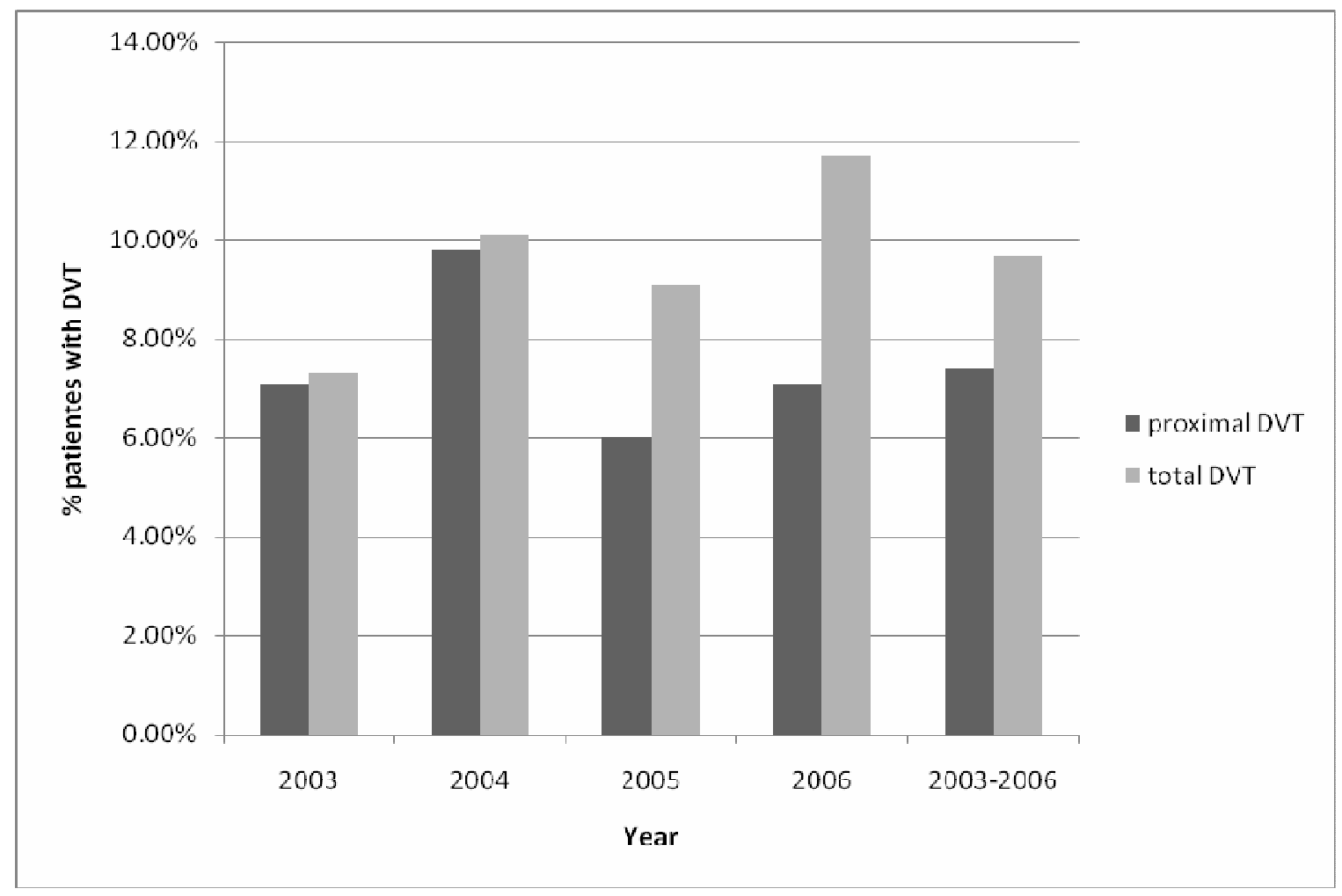


Table 2 Incidence of lower extremity DVT

\begin{tabular}{|l|c|c|c|c|c|}
\hline & $\mathbf{2 0 0 3}$ & $\mathbf{2 0 0 4}$ & $\mathbf{2 0 0 5}$ & $\mathbf{2 0 0 6}$ & \multicolumn{1}{c|}{ Total } \\
\hline Patients with lower extremity DVT & $7.3 \%$ & $10.1 \%$ & $9.1 \%$ & $11.7 \%$ & $9.7 \%$ \\
\hline Patients with proximal DVT & $7.1 \%$ & $9.8 \%$ & $6.0 \%$ & $7.1 \%$ & $7.4 \%$ \\
\hline Patients with distal only DVT & $0.2 \%$ & $0.3 \%$ & $3.1 \%$ & $4.6 \%$ & $2.3 \%$ \\
\hline
\end{tabular}


Table 3 Primary diagnosis among DVT patients

\begin{tabular}{|l|c|c|}
\hline Primary Diagnosis & Total patients & Percent patients \\
\hline SAH & 115 & $48.5 \%$ \\
\hline ICH & 28 & $11.8 \%$ \\
\hline Malignancy & 26 & $11.0 \%$ \\
\hline Unruptured aneurysm/AVM & & $8.9 \%$ \\
\hline Mass/tumor $^{1}$ & 21 & $4.6 \%$ \\
\hline Other $^{2}$ & 11 & $15.2 \%$ \\
\hline
\end{tabular}

1 Arteriovenous malformation, cerebrovascular anomaly

2 Most common diagnoses were infection, hydrocephalus, mental status change, and seizure. 